Macedonian Pharmaceutical Bulletin, 66 (Suppl 1) 115 - 116 (2020)

Online ISSN $1857-8969$

UDC: $615.277 .015: 546.26-022.532$

DOI: 10.33320/maced.pharm.bull.2020.66.03.057

Short communication

\title{
Effect of irradiation on the physicochemical and biopharmaceutical properties of Temozolomide loaded carbon nanotubes
}

\author{
Radmila Ilijeva $^{1}$, Anita Grozdanov ${ }^{2}$, Piotr Ulański ${ }^{3}$, Slawomir Kadlubowski ${ }^{3}$, \\ Marija Runceva ${ }^{1}$, Ana Ivceska $^{1}$, Kristina Mladenovska ${ }^{1}$ \\ ${ }^{1}$ Faculty of Pharmacy, Ss. Cyril and Methodius University, 1000 Skopje, N. Macedonia \\ ${ }^{2}$ Faculty of Technology and Metallurgy, Ss. Cyril and Methodius University, 1000 Skopje, N. Macedonia \\ ${ }^{3}$ Institute of Applied Radiation Chemistry, Lodz University of Technology, Lodz, Poland
}

\section{Introduction}

The distinct properties of carbon nanotubes (CNTs), in particular high surface area (that provides high drug loading), intrinsic optical and thermal properties (that enable multimodal real-time tracking and photo-thermal applications), propensity to functional modification and biocompatibility and nanofluid nature make them useful for controlled brain (tumor) drug delivery. By attachment of reactive functional groups, basic electrical, optical and physicochemical properties can be changed and conjugation of ligands and drugs to the surface of CNTs enhanced. Their capacity to cross the bloodbrain-(tumor)-barrier by energy-dependent and independent mechanisms (needle-like crossing) is another major advantage. Due to the electrical conductive capacity, strong mechanical properties and morphology similar to those of neurons, CNTs satisfy the requirements for successful neurorestoration, genesis and plasticity. Also, the structure and dimensions of CNTs resemble those of certain neuronal structures and elements relevant for physiological activity and processing of neuronal information (Guo et al., 2017). Having all this in regard, the aim of the study was to prepare and characterize Temozolomide (Tem) loaded multiwalled CNTs (MWCNTs-Tem). In addition, the effect of irradiation on physicochemical and biopharmaceutical properties of MWCNTs-Tem (IMWCNTs-Tem) was evaluated. Tem is a first line alkylating and radio-sensitizing agent used for treatment of anaplastic astrocytoma and glioblastoma multiforme (GBM).

\section{Materials and Methods}

MWCNTs were prepared by an original procedure of reduction of Li molten salts on graphite cathode (Dimitrov et al., 2013). Solution of Tem was added to MWCNTs suspension in $0,1 \mathrm{M} \mathrm{HCL}$ (MWCNTs:Tem 1:3), ultrasonicated for $1 \mathrm{~h}$ and stirred for $96 \mathrm{~h}$. Afterwards, the MWCNTs-Tem were isolated by ultracentrifugation $(12000 \mathrm{rpm} / \mathrm{min}, 15$ min, Eppendorf MiniSpin, Germany), rinsed $3 \times$ with double-distilled water and dried at room temperature. Encapsulation efficacy (EE) was determined as a difference between the total amount of Tem in the initial solution and filtrate. The drug content (DC) was calculated as a ratio between the total amount of loaded Tem and MWCNTs-Tem. The concentration of Tem was measured with

\footnotetext{
* radmila.ilijeva@gmail.com
} 
UV/VIS spectrometer $(\lambda=328 \mathrm{~nm}$, UV/VIS Perkin Elmer Lambda 16, USA). Particle size distribution and zeta potential were determined by NanoZS-100 (Malvern Instruments Ltd., UK), after dilution of (I-) MWCNTs-Tem in PBS, pH $7.4(0,0001 \mathrm{M})$ with Polysorbate $^{\circledR} 20(2 \%$ (w/w), Sigma-Aldrich, UK). In vitro Tem release from (I-)MWCNTs-Tem was followed through a dialysis membrane (Mw cut-off 12000, Sigma-Aldrich) in PBS pH $7.4\left(37^{\circ} \mathrm{C} \pm 2{ }^{\circ} \mathrm{C}\right.$, $50 \mathrm{rpm} / \mathrm{min})$. At predetermined time intervals, samples were collected and concentration of released Tem was estimated. The release data were fitted into various models to determine the release kinetics. To find out the mechanism of drug release, $60 \%$ of drug release data was fitted in the Korsmeyer-Peppas (KP) model. Samples have been irradiated by electron beam generated by ELU-6 linear accelerator (Elektronika, Russia), the absorbed dose being 50 kGy. Pulses of $6 \mathrm{MeV}$ electrons (pulse duration: 4 $\mathrm{ms}$ ) have been delivered at a frequency of $20 \mathrm{~Hz}$, leading to an average dose rate of $5 \mathrm{kGy} / \mathrm{min}$ (as determined by alanine dosimetry).

\section{Results and Discussion}

EE of MWCNTs-Tem was $62.0 \pm 3.5 \% \quad(n=6)$, while the DC $16.0 \pm 1.2 \%(\mathrm{n}=6$, theor. content $25 \%$ ). Statistically significant differences were observed between the MWCNTs-Tem and I-MWCNTs-Tem in surface charge $(-13.3 \pm 2.3 \mathrm{mV}$ and $-1.2 \pm 0.3 \mathrm{mV}$, accordingly, $\mathrm{n}=6)$ and $\mathrm{d}_{50} / \mathrm{PDI}(145 / 0.385$ and 654/0.628, accordingly, $\mathrm{n}=6$ ), suggesting more homogeneous size distribution of MWCNTs-Tem and formation of aggregates of I-MWCNTs that affect surface charge. Literature data point that exposure of MWCNTs on gamma-irradiation at lower doses (25 and $50 \mathrm{kGy}$ ) improved the graphitic order of MWCNTs, higher doses (100 and $150 \mathrm{kGy}$ ) introduced structural imperfection and at very high dose (200 kGy), the structure become distorted (Silambarasan et al., 2017). In a study of Jun et al. (2010), increased release rate of cancer ancillary drug tea polyphenols from chitosan-MWCNTs was observed by gamma irradiation. The opposite was observed in this study, where statistically significant difference in Tem release kinetics between MWCNTs-Tem and I-MWCNTs-Tem was observed. The cumulative percent of Tem released after $2 \mathrm{~h}$ and 9 days was higher $(22 \pm 6 \%$ and $85 \pm 5 \%)$ from MWCNTs-Tem than I-MWCNTs-Tem $(15 \pm 2 \%$ and $58 \pm 16 \%(\mathrm{n}=3)$, accordingly), which can be explained by higher particle size of I-MWCNTsTem. Higuchi model was the best fit model for the Tem release $\left(\mathrm{R}=0.926\right.$ and $\mathrm{R}=0.957, \mathrm{k}=4.04 \mathrm{~h}^{-1}$ and $2.53 \mathrm{~h}^{-1}$, accordingly). The exponent " $\mathrm{n}$ " in the K-P model was $\leq 0.45$ for both (I-) MWCNTs-Tem, indicating Fickian diffusion in the drug release.

\section{Conclusion}

The paper demonstrates the potential of MWCNTs for delivery of Tem to tumor cells (incl. brain tumors), based on the basic physicochemical and biopharmaceutical properties. Incorporation of Tem into MWCNTs is simple and the content of Tem in the prepared MWCNTs is relatively high. The size of MWCNT-Tem is suitable for delivery via the $\mathrm{BB}(\mathrm{T}) \mathrm{B}$, although there are literature data suggesting that cell accumulation is non-dependent on length/size of the CNTs (SWCNT and MWCNT with average size between 195 and $630 \mathrm{~nm}$ ). Surface charge is also suitable for effective delivery in tumor cells, having in regard that slightly negative or neutral charge is required in central circulation. Tem showed low potential for early release from MWCNTs (and thus low potential for systemic toxicity) and potential for continuing release at the site of action, having in regard the $\mathrm{pH}$ of brain tumor cells (e.g. $\mathrm{pH}$ of GBM 7.2). The exposure to irradiation has affected the physicochemical and biopharmaceutical properties of MWCNTs-Tem.

\section{References}

Dimitrov, A.T., Tomova, A., Grozdanov, A., Popovski, O., Paunovic, P., 2013. Electrochemical production, characterization, and application of MWCNTs, J. Solid State Electrochem. 17, 399-407.

Guo, Q., Shen, X., Li, Y., Xu, S., 2017. Carbon nanotubes-based drug delivery to cancer and brain. Curr. Med. Sci. 37, 635-641.

Jun, L., Hao, S., Yao-Dong, D., 2010. Using gamma radiation from drug releasing from MWNT vehicle. Chinese Phys. Lett. 27, 38-104.

Silambarasan, D., Surya, V.J., Iyakutti, K., Vasu, V., Asokan, K., Kawazoe, Y., 2017. Gamma (gamma)ray irradiated multi-walled carbon nanotubes (MWCNTs) for hydrogen storage. Appl. Surf. Sci. $418,49-55$.

Maced. Pharm. Bull. 66 (Suppl 1) 115 - 116 (2020) 